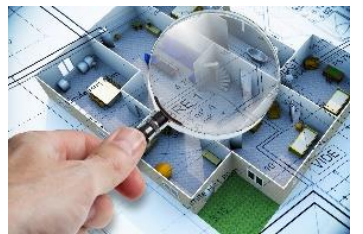

\title{
A study of soil vibro-compaction in medium sands
}

\author{
Norbert KUREK ${ }^{1}$ \\ Karolina TRYBOCKA ${ }^{1}$ \\ ${ }^{1}$ Menard Ltd., 44c Powąkowska Str., 01-797 Warsaw, Poland \\ corresponding author: ktrybocka@menard.pl
}

\begin{abstract}
The present article offers an analysis of the vibroflotation technique at a test plot set up in central Poland. The role of the test plot was to examine and evaluate the effectiveness of deep ground compaction and some selected parameters by applying vibroflotation activities as well as to verify the impact of vibration propagation on the existing structures. Below are then presented selected results concerning vibration measurements and geotechnical in-situ tests.
\end{abstract}

Keywords: vibroflotation; compaction; vibrations; vibration measurements; in-situ tests.

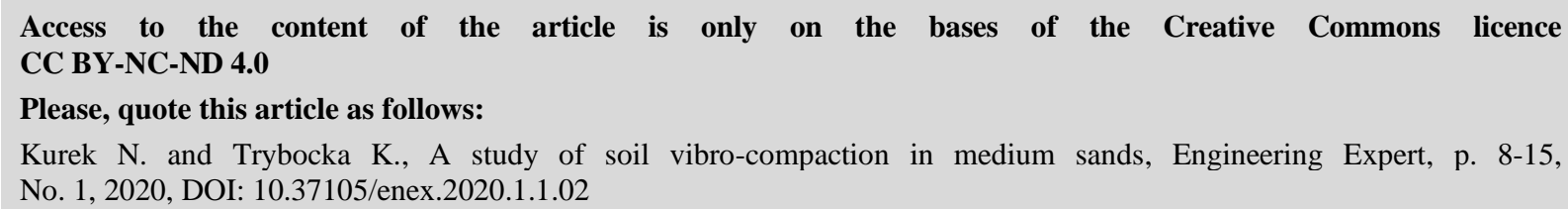

\section{Introduction}

Vibroflotation is deep compaction technique used for non-cohesive soils which is performed by means of a vibroflot, which vibrates horizontally. Under such cyclic vibrations that propagate in the ground, the water pressure in the ground pores rises as well. This leads initially to a local liquefaction of the soil medium but then causes the soil grains to move towards their tighter arrangement. This results in an overall reduction of the pore volume in the soil of the improved area, and so the material is allowed to densify.

That vibration method can be used in medium and coarse sands with a content of particle size of not more than $0.063 \mathrm{~mm}$ less than $10 \%$, content of particles $\leq 0.002 \mathrm{~mm}$ less than $2 \%$ and a uniformity coefficient $C_{u}>2.5$. The fines content in the compacted soil is a key parameter for the applicability of this ground improvement method. Due to high fines content, the shear stresses generated by the vibrator are not transmitted that effectively and the vibrations are considerably damped [1]. The movement of particles, necessary for compaction, is also limited [2].

\section{Description of geological conditions}

The results of the study and the measurements dealt within this article come from a site in central Poland, where vibroflotation method was used for soil deep compaction. There are mainly medium and coarse sands interwoven with layers of fine sands in the site-specific subsoil stratum. The $I_{D}$ density index of the sands ranged from 0.25 to 0.75 in their original state. And it grew for coarsegrained soils with greater depth. An additional increase of $I_{D}$ was observed under the groundwater 
table. The water table was located at the level of approx. $4.5 \mathrm{~m}$ below the level of the working platform, as seen on the schematic geological cross-section in Fig. 1.

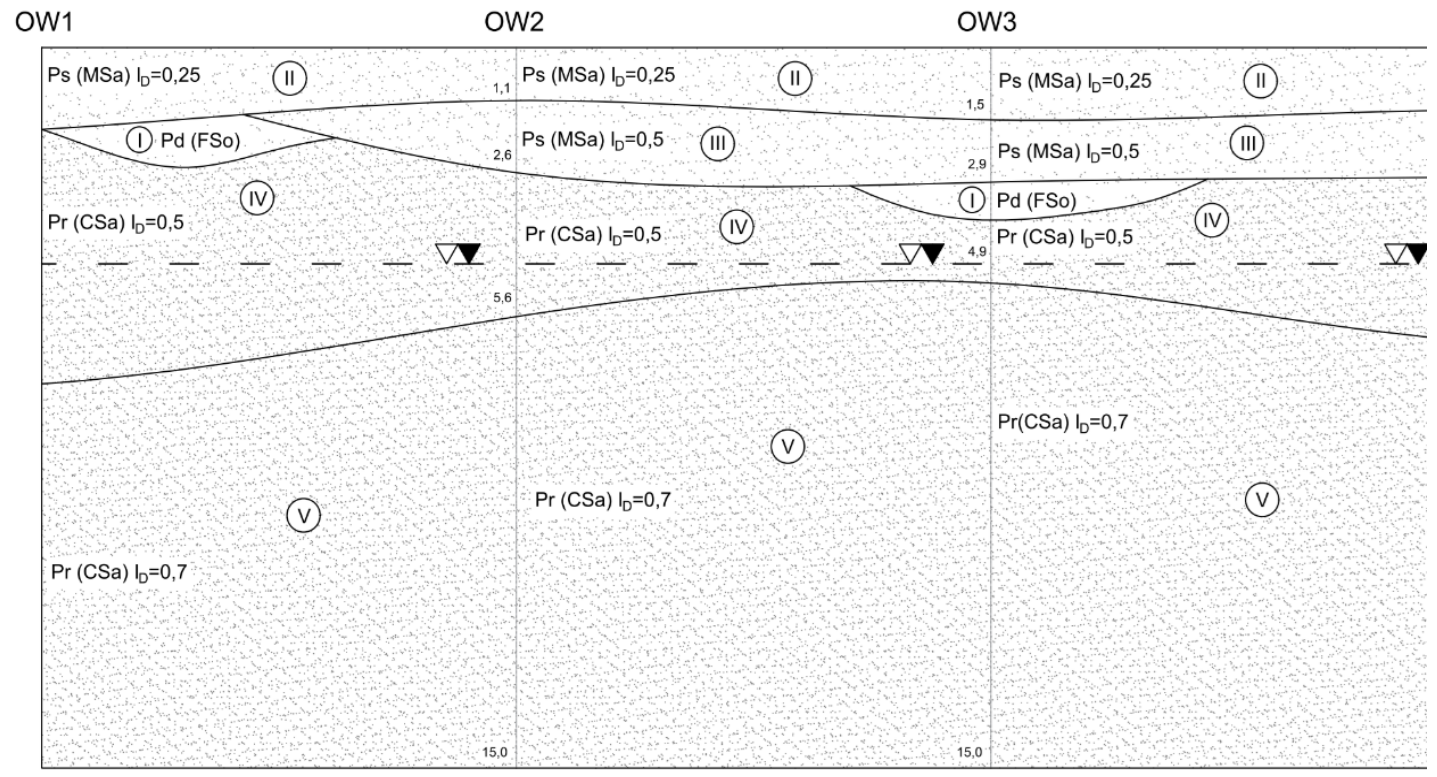

Fig. 1. Geological cross-section in the improved area.

The uniformity coefficient $C_{u}=d_{60} / d_{10}$ ranged from 3 to 5 . A comparison of the grain-size distribution curves for the soil from the test plot is presented in Fig. 2. That diagram, by and courtesy of Menard Group, presents four zones of granulometry, which classify soils in terms of their susceptibility to dynamic compaction. The soil samples from the test plot are all compactible or ideally compactible. The curves closest to the ideal shape were for the samples from the depth of $3.5 \mathrm{~m} ; 5.0$ $\mathrm{m} ; 7.5 \mathrm{~m}$, while the curves from the depth of $1.5 \mathrm{~m} ; 13.5 \mathrm{~m} ; 9.5 \mathrm{~m}$ had a smaller uniformity coefficient and a lower content of particles with a size greater than $1 \mathrm{~mm}$. The juxtaposition of samples from between 2.0 and $7.5 \mathrm{mbgl}$ and of their results before and after vibroflotation showed ideal suitability for soil compaction (shown later herein).

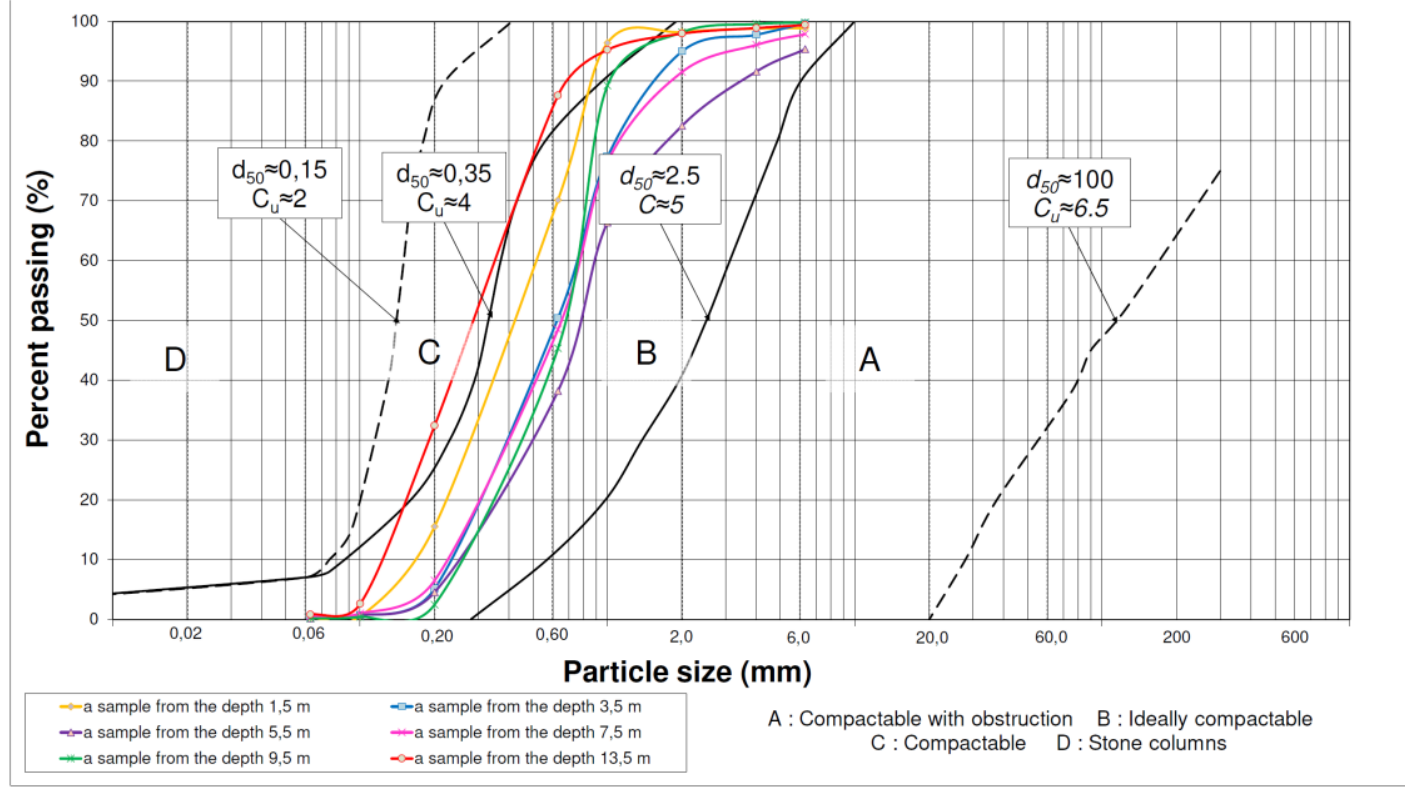

Fig. 2. Comparison of the grain-size distribution curves of the test plot. 


\section{Deep compaction method}

The soil compaction at the site analyzed was carried out with a vibroflot freely suspended from a crane. The type of the vibrator used was the V23 model, with power rating of $130 \mathrm{~kW}$, frequency of 30 $\mathrm{Hz}$ and vibration amplitude of $23 \mathrm{~mm}$. Vibroflotation points were arranged in a triangular grid system with a side length of the equilateral triangle of approx. $2.8 \mathrm{~m}$. The depth of deep compaction in relation to the level of the working platform was approx. $6 \div 7 \mathrm{~m}$. No additional backfill material was used during the compaction but only the potential of the subsoil material was exploited.

It should be noted that the vibroflotation technique is not effective in the near-surface zone, that is up to the depth of approx. $1 \mathrm{~m} \div 1.5 \mathrm{~m}$ below the working platform level. This phenomenon is caused by low effective stresses in the subsoil layer. The particular depth from which the compaction effect occurs depends on many factors, including the type of soil, its volumetric weight, possible additional soil load, frequency and amplitude of vibrations. Loosening of the soil may occur in the subsoil zone. Additionally, reduction in the volume of pores in the soil medium causes its compaction, which results in subsiding of the ground level. Allowing for these phenomena it is recommended to form a working level for the vibroflotation technique at approx. $1.5 \div 2 \mathrm{~m}$ above the foundation level of the future structure or to apply additional treatments to densify the subsoil layer.

\section{Deep compaction method}

Appropriate measurements were taken at the test plot to assess the effectiveness of the technique applied there. Both before and after the compaction, tests were made with a static probe (CPTU), dilatometer tests (DMT) and geodetic measurements of the working platform level. The postcompaction probe soundings were located at the intersection of the middle triangles formed of vertices that were the compaction points. The vibroflotation process caused the working platform to subside in the test plot by approx. $50 \mathrm{~cm}$ on average, which constitutes approx. $8 \%$ of the thickness of the compacted layer.

The results of CPTU static soundings before and 7 days after the compaction process and for the $2.8 \mathrm{~m}$ spacing of compaction points are shown in Fig. 3. The comparison of the measurement results clearly shows a significant increase in the resistance on the $q_{c}$ cone. On average this value doubled in terms of the vibroflot work depth. The $I_{D}$ index of soil strengthening defined in accordance with the formula as suggested in the publication by Dove et al. [9] was on average:

$$
I_{D}=\frac{q_{c, \text { after }}}{q_{c, \text { before }}}-1 \approx 1
$$

where:

$q_{c, \text { after }}$ - cone resistance value after vibroflotation;

$q_{c, \text { before }}$ - cone resistance value before vibroflotation.

As expected, up to a depth of $1.5 \mathrm{~m}$ below the working platform level no strengthening effect was obtained, as explained above.

According to DeJong et al. [8] the fs sleeve friction is a parameter that is less sensitive to changes in soil compaction caused by vibroflotation and is not a reliable parameter for the assessment of the state of a particular soil. A slight fall in the value of the $f_{s}$ sleeve friction was observed at the test plot. 


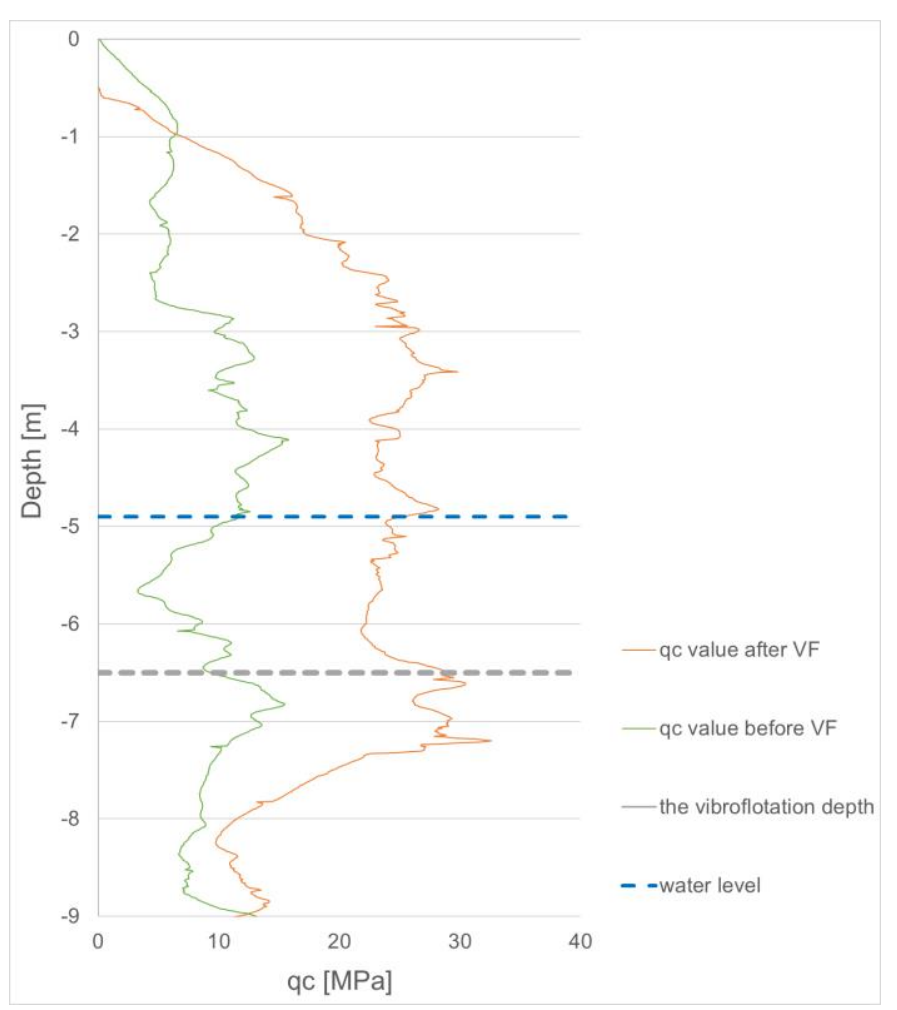

Fig. 3. The results of tests with a static probe before and after compaction.

The results obtained by dilatometer are juxtaposed in Fig. 4. The values of the $K_{d}$ horizontal stress coefficient and the $E_{D M T}$ dilatometer modulus show a greater than twofold increase after performing deep compaction. The increase in the angle of internal friction in the compacted zone is estimated to be 5 degrees on average.
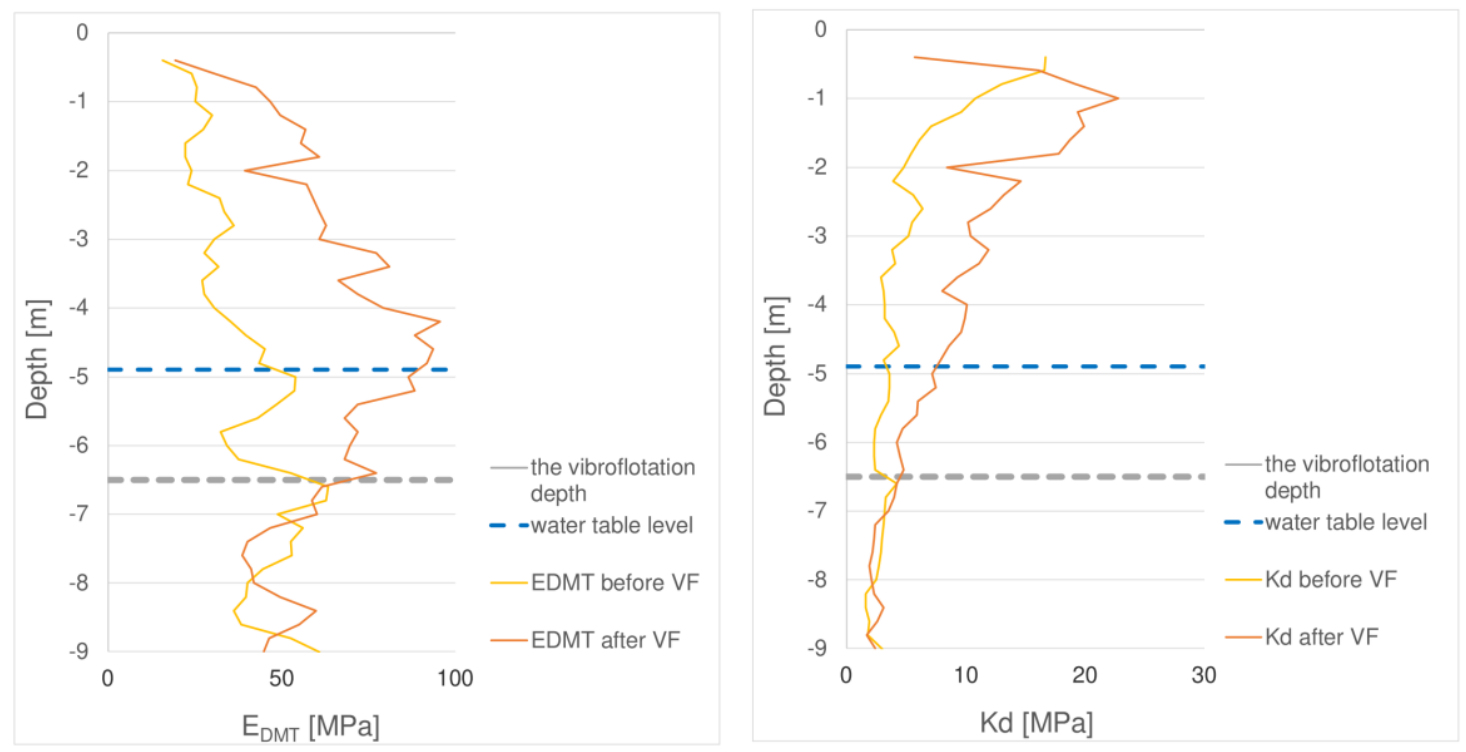

Fig. 4. The results of dilatometer tests before and after compaction.

Compared to other test plots [3], [10], [11], the general results do not differ significantly and can be considered reliable in terms of applying that technology in similar ground conditions. It should be emphasized that, according to the literature and other measurements in other inspection plots, that 
technique offers the possibility of obtaining much higher densities, such as required for the improved area. In the example presented here, no additional backfill material was used during its compaction.

\section{Measurements of vibrations}

In order to measure the propagation of vibrations in the soil-water medium there were used sound and vibration level meters with transducers, a mechanical vibration calibrator, equipment, cabling and appropriate software. The vibrations were recorded on the three main axes: $\mathrm{X}$ and $\mathrm{Y}$ (horizontal directions) and $Z$ (vertical direction). Peak vibration velocities (PPV) were recorded in $\mathrm{mm} / \mathrm{s}$. The vibration monitoring sensors were spaced in three configurations:

- Vibration measurements were conducted at even distances: four vibration sensors placed on concrete sleepers at the following distances from the site: $5 \mathrm{~m}, 10 \mathrm{~m}, 20 \mathrm{~m}$ and $50 \mathrm{~m}$ (Fig. 5) which is the test plot.

- Measurements of vibrations of existing structures: railway embankment (28 m), rain drainage chamber $(11 \mathrm{~m})$, reinforced concrete wall of a warehouse $(18 \mathrm{~m})$, foundation of a flyover $(50 \mathrm{~m})$.

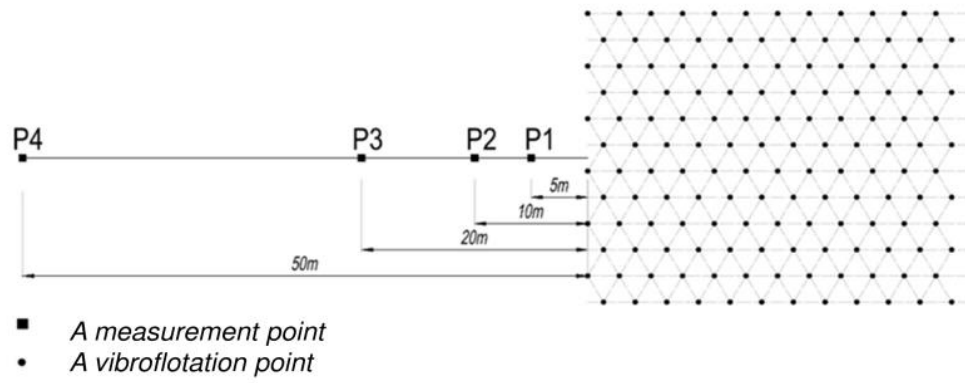

Fig. 5. Spacing of measurement points in the test plot.

\section{Measurement results and their analysis}

An example diagram of the recorded vibration velocities per time is presented below (Fig. 6).

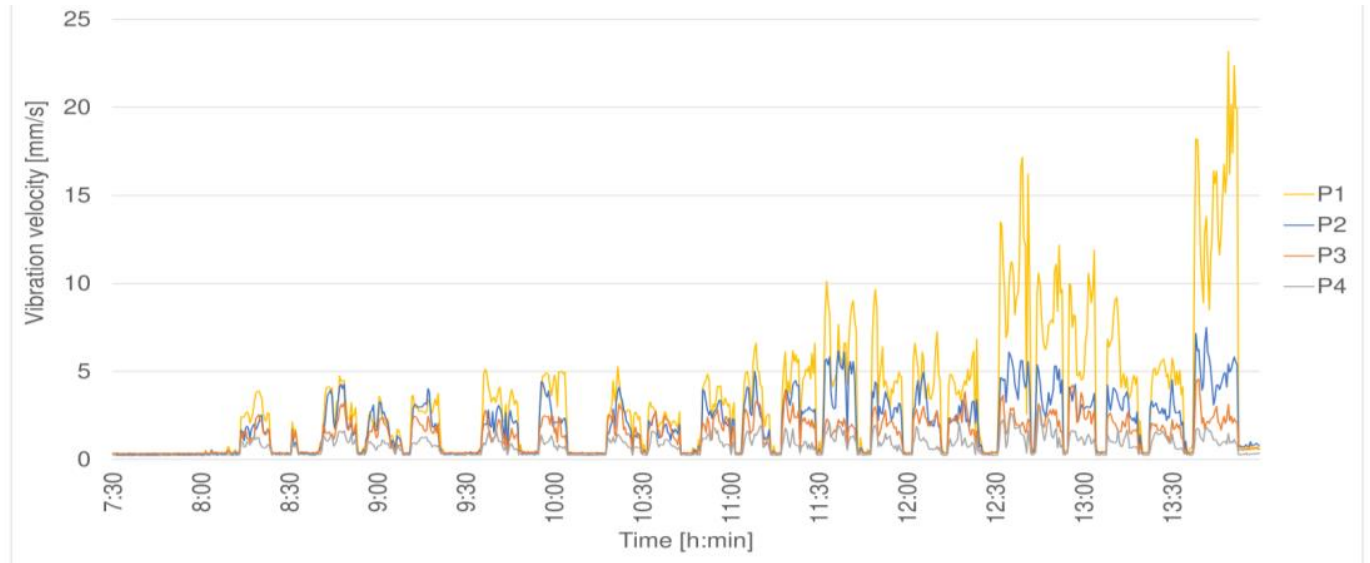

Fig. 6. An example graph of the measurements of resultant vibration velocity for the test plot. Measurements taken at the following distances: P1-5m, P2-10m, P3-20m, P4-50m.

A Polish national standard [5] determines the response of a building to kinematic excitation. This standard recommends a detailed analysis of structures in the spatial model of a building (FEM) reading that: Forces of inertia and the resulting displacements and stresses in the elements 
of an existing structure should be determined in the model of a building according to the principles of dynamics of buildings and at the same time assuming the kinematic excitation. This standard also offers simplified methods of dynamic analysis and the scale of dynamic impacts on masonry construction as well as buildings made of large-scale prefabricated components.

For information purposes, Fig. 7 and Fig. 8 refer to German [6] and French [7] national standards, respectively, which may be of practical use in the preliminary analysis phase.

Fig. 7 shows the graph of vibration velocity and their frequency in relation to the benchmark curves L1 (office buildings, industrial buildings), L2 (residential buildings), L3 (structures sensitive to vibrations) in accordance with the German national standard [6], for a point located at a distance of $20 \mathrm{~m}$ from vibroflotation (P3).

Fig. 8 shows the graph of vibration velocity and its frequencies in relation to the curves under the French standard [7] for structures resistant, sensitive and very sensitive to vibrations.

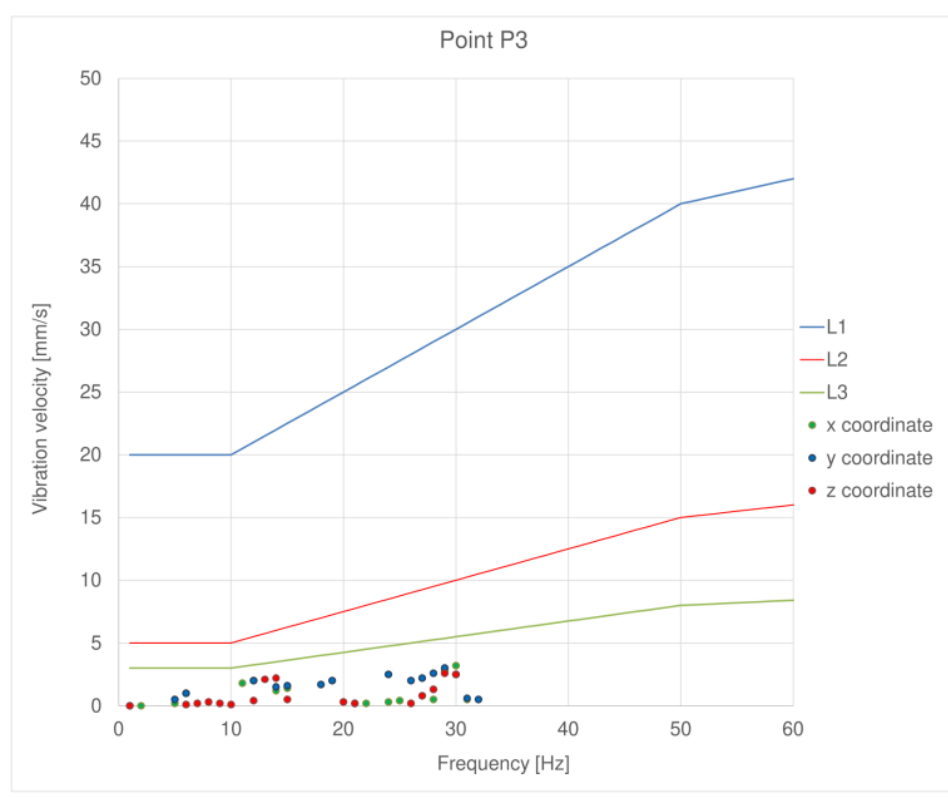

Fig. 7. Graph of vibration velocity and its frequencies in relation to curves L1, L2, L3 in accordance with DIN 4150-3. Measurements taken for the P3 point at a distance of $20 \mathrm{~m}$ from the source of vibrations.

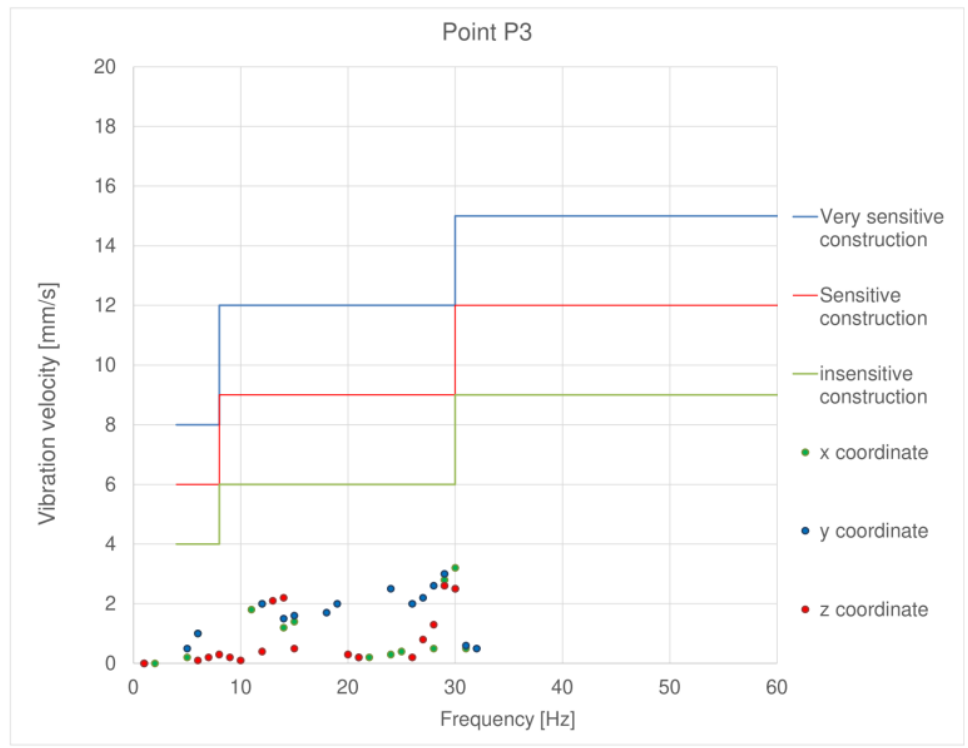

Fig. 8. Graph of the vibration velocity and its frequencies in relation to the French national standard. Measurements taken for the $\mathrm{P} 3$ point at a distance of $20 \mathrm{~m}$ from the vibration source. 


\section{Presentation of analyses}

Fig. 9 shows the peak values of the vibration velocity (PPV) (resultant vectors) as a function of the distance from the centre of vibroflotation. The dashed line shows the generalized formula describing the value of the maximum vibration velocity in terms of the distance from the vibration source. The following constants were adopted for the reference function: $\mathrm{K}=1.5 ; \mathrm{x}=1$; $\mathrm{E}$ - vibration energy value; $\mathrm{R}$ - distance from the source of vibration.

It should be noted that due to our simultaneous performance of other work and the movement of vehicles in the vicinity of the test plot, the measurements also reflect the impact from other vibration sources. However, measurements recorded as a result of violent wind gusts were excluded from the analysis.

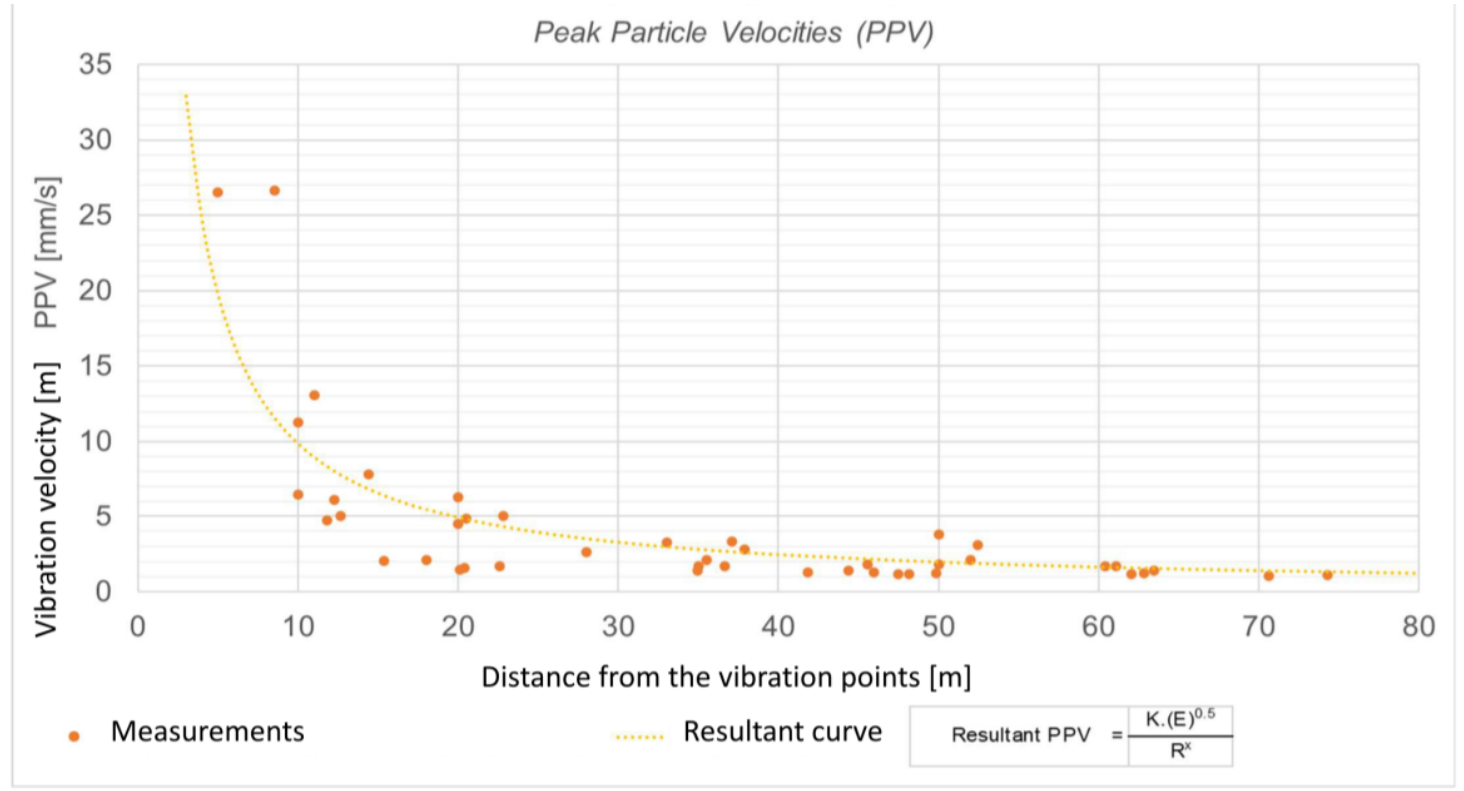

Fig. 9. Graph of recorded PPV (resultant values from three directions $X, Y, Z$ ) in terms of the distance from the centre of vibroflotation.

Vibration velocities decrease exponentially along with the distance from the source of vibrations. In these site-specific ground conditions and at a distance greater than $10 \mathrm{~m}$ from the vibration source, no vibration velocity values were observed to be higher than $25 \mathrm{~mm} / \mathrm{s}$. At a distance of more than 20 $\mathrm{m}$ from the vibration source the measured PPV values were less than $7 \mathrm{~mm} / \mathrm{s}$.

Taking into account the vibration frequency from $0 \mathrm{~Hz}$ to $30 \mathrm{~Hz}$ the vibration velocity did not exceed the values allowed for office and industrial buildings according to DIN 4150-3 (PPV $<20 \mathrm{~mm} / \mathrm{s}$ ) at all the measuring points at a distance equal to or greater than $10 \mathrm{~m}$. There were no residential buildings or similar existing structures in the area of improvement. However, conclusions can be drawn from the tests that with such ground conditions, buildings such as those mentioned above and located at a distance of more than $25 \mathrm{~m}$ would be in a safe zone in terms of vibration propagation. The distance of $60 \mathrm{~m}$ can be regarded as a safe distance for structures sensitive to vibrations, because the PPV values do not exceed $2 \mathrm{~mm} / \mathrm{s}$.

\section{Conclusions}

The article presents the results of selected tests and measurements, which for this particular test plot give an idea of the effectiveness and potential impact of the vibroflotation technique on the existing surrounding structures. 
Vibroflotation activities belong to a group of subsoil strengthening techniques, for which it is recommended at each time to carry out measurements of test plots and then select technical and geometric parameters appropriate for a given area. In addition, it is also recommended to carry out vibration propagation measurements in the case of existing buildings located between $30 \mathrm{~m}$ and $50 \mathrm{~m}$ (depending on the sensitivity of the structure) off the site under improvement.

The article presents the results of the vibration velocity measurements for the waves generated and propagating during the deep compaction of non-cohesive soils while using the vibroflotation method. The measured vibration values were compared with the example permissible values. When analyzing the possibility of using vibration techniques, it should be taken into account that during works a propagating wave causes compaction of soil in its adjacent area. In the case of expected excessive vibrations or settlements of the ground it is necessary to modify the technical parameters or apply solutions that will damp vibrations. Despite the need to calibrate and verify the vibroflotation activities at each area of improvement due to its site-specific ground conditions it should be emphasized that this technique may turn out to be economic, fast and effective.

\section{Bibliography}

[1] R. Hitchman. An Evaluation of the Phoenix Machine: A New Apparatus for the In-Situ Densification of Soil. A master's thesis, The University of British Columbia, 1989.

[2] F.G. Bell. Engineering Treatment of Soil. London E \& FN Spon, 1993.

[3] N. Kurek. Quality Control of Deep Compaction of Non-Cohesive Soils. A doctoral dissertation, Gdańsk: Gdańsk University of Technology, 2013.

[4] Hamidi, B., Varaksin, S. and Nikraz, H., 2011. A Case of Vibro Compaction Vibration Monitoring in a Reclaimed Site. International Conference on Advances in Geotechnical Engineering (ICAGE 2011), pp. 861-866.

[5] Polish Standard PN-B-02170: 2016-12: Assessment of the Harmfulness of Vibrations Transmitted by the Ground onto Buildings.

[6] German Standard DIN 4150-3: 1999-02: Structural vibration. Part 3, Effects of vibration on structures.

[7] Regulation "Circulaire du 23/07/86 relative aux vibrations mécaniques émises dans l'environnement par les installations classées pour la protection de l'environnement" A French regulation.

[8] J.T.DeJong et al. "Effect of Surface Texturing on CPT Friction Sleeve Measurements" in: Journal of Geotechnical and Geoenvironmental Engineering, pp. 158-168, February 2001.

[9] J.E. Dove et al. “A CPT-Based Index for Evaluating Ground Improvement” in: GeoDenver, 2000, pp. 296-310, Denver (Colorado), American Society of Civil Engineers, August 2000.

[10] D. Berthier et al. "Marine and Land Based Compaction Works At The Port Botany Project" in: Ground Improvement Technologies and Case Histories, pp. 221-229, Sydney, January 2009.

[11] Wang D., Chen S., Liang X., Zhou H. (2018) "Application of Cone Penetration Test Technology in Whole Process Inspection of Reinforcing Hydraulic Fill Sand Foundation" in: Li L., Cetin B., Yang X. (eds) Proceedings of GeoShanghai 2018 International Conference: Ground Improvement and Geosynthetics, pp. 145-156. GSIC 2018. Springer, Singapore. 